\title{
Componentes da Produção de Forragem em Pastagens dos Capins Tanzânia e Mombaça Adubadas com Quatro Doses de NPK ${ }^{1}$
}

\author{
Danilo Gusmão de Quadros ${ }^{2}$, Luís Roberto de Andrade Rodrigues ${ }^{3}$, Vanildo Favoretto ${ }^{3}$, \\ Euclides Braga Malheiros ${ }^{4}$, Valdo Rodrigues Herling ${ }^{5}$, Allan Kardec Braga Ramos ${ }^{6}$
}

\begin{abstract}
RESUMO - O trabalho foi conduzido na FCAV-Unesp, em Jaboticabal-SP, para avaliar os efeitos de quatro doses combinadas de NPK sobre os componentes da produção e das perdas de matéria seca (MS) em pastagens dos cultivares Tanzânia e Mombaça de Panicum maximum Jacq., manejadas com 28 dias de descanso e com altura média de $30 \mathrm{~cm}$ de resíduo pós-pastejo. As avaliações foram realizadas em parcelas de $96 \mathrm{~m}^{2}$ segundo um fatorial 2 (cultivares) x 4 (doses de NPK), em blocos completos ao acaso, com três repetições. As adubações estudadas corresponderam ao decréscimo em $30 \%$ e aos acréscimos em 30 e $60 \%$ de uma dose "padrão" com 145; 21,6 e $180 \mathrm{~kg} /$ ha de N, $\mathrm{P}_{2} \mathrm{O}_{5}$ e $\mathrm{K}_{2} \mathrm{O}$, respectivamente (referente a 1,2; a 0,08; e a $1,2 \%$ de N, P e K na MS, com produção estimada em 12000 kg/ha). A MS verde (MSV) em pré-pastejo e no resíduo aumentou linearmente com a adubação, com maiores valores obtidos para o cv. Mombaça (9183 e $5227 \mathrm{~kg} / \mathrm{ha}$, respectivamente) do que para o cv. Tanzânia (6275 e 3808 kg/ha, respectivamente). A participação de lâminas foliares na MSV em pré-pastejo foi menor no cv. Tanzânia (51 \%) do que no cv. Mombaça (54\%). A densidade de perfilhos não variou com a adubação. O aumento do peso de perfilho com a elevação das doses de NPK resultou em maiores produções de MSV. A MS senescida (média de $3108 \mathrm{~kg} /$ ha de MS) não diferiu entre cultivares. De modo geral, quanto maior a dose de adubo aplicado, maiores foram a taxa de acúmulo diário de MSV e a MSV perdida por pisoteio. O cv. Mombaça apresentou maior potencial de resposta à adubação do que o cv. Tanzânia, com taxas de lotação de 6,2 e 4,0 UA/ha, respectivamente.
\end{abstract}

Palavras-chave: adubação de pastagens, Panicum maximum, perdas por pisoteio, taxa de acúmulo de MSV/ha/dia, UA/ha

\section{Components of Herbage Production of Tanzania and Mombaça Pastures Fertilized with Four Doses of NPK}

\begin{abstract}
The experiment was conducted at the FCAV-Unesp, Jaboticabal-SP, to evaluate the effects of the fertilization with four doses of NPK on the components of dry matter (DM) and losses by trampling of two cultivars of Panicum maximum Jacq. (Tanzania and Mombaça) under grazing, having a rest period of 28 days and an average height of residue of $30 \mathrm{~cm}$. A complete randomized block design was used with treatments arranged in a $2 \times 4$ factorial with three field replications. The fertilization doses corresponded to the reduction of $30 \%$ and the increase of 30 and $60 \%$ in relation to a standard dose of $145 ; 21.6$; and $180 \mathrm{~kg} / \mathrm{ha}$ of $\mathrm{N}_{2} \mathrm{P}_{2} \mathrm{O}_{5}$, and $\mathrm{K}_{2} \mathrm{O}$, respectively (assuming the contents of 1.2, 0.08 , and $1.2 \%$ of N, P, and K in DM, to reach an estimated DM production of $12000 \mathrm{~kg} / \mathrm{ha}$ ). There was a linear effect of fertilization doses on green DM (GDM) before and after grazing. The cv. Mombaça exhibited higher herbage mass before and after grazing (9183 and $5279 \mathrm{~kg} / \mathrm{ha}$ of GDM, respectively) than the cv. Tanzania ( $6275 \mathrm{and} 3808 \mathrm{~kg} / \mathrm{ha}$ of GDM, respectively). The proportion of leaf blade in the GDM available was lower in the cv. Tanzania (51\%) than in the cv. Mombaça (54\%). The tiller density was not affected by the fertilization doses. However, the increase in tiller weight due to fertilizer doses was responsible for the higher GDM production. The senesced DM did not vary between cultivars, with a mean value of $3108 \mathrm{~kg} / \mathrm{ha}$. In general, higher rates of fertilization resulted in greater GDM accumulation rate and higher losses of GDM by trampling. The cv. Mombaça showed a greater response potential to fertilization than the $\mathrm{cv}$. Tanzania with stocking rates of 6.2 and $4.0 \mathrm{UA} / \mathrm{ha}$, respectively.
\end{abstract}

Key Words: AU/ha, green DM accumulation, DM losses by trampling, Panicum maximum, pasture fertilization

\section{Introdução}

A intensificação do uso de pastagens tropicais para a produção de ruminantes tem sido cada vez mais freqüente. Para alcançar alta produtividade animal há necessidade de adubações de formação e de manutenção das pastagens, além da escolha de gramíneas forrageiras que possuam potencial para produção de forragem com bom valor nutritivo.

As gramíneas do gênero Panicum exigem solos de média a alta fertilidade para uma boa produção de forragem (Alcântara et al., 1993). A grande diversidade de tipos de solos do Brasil, em relação à sua fertilidade natural, influencia a produção de forra-

\footnotetext{
${ }^{1}$ Parte da Dissertação do primeiro autor para obtenção do título de Mestre em Zootecnia na FCAV-UNESP, com bolsa da CAPES

${ }^{2}$ Aluno de Pós-Graduação em Zootecnia, FCAV - UNESP, 14884-900, Jaboticabal-SP. Bolsista do CNPq. E.mail: quadros@fcav.unesp.br

${ }^{3}$ Prof. do Depto. de Zootecnia da FCAV - UNESP. E.mail: Irodrigs@fcav.unesp.br

4 Prof. do Depto. de Ciências Exatas da FCAV- UNESP. E.mail: euclides@fcav.unesp.br

${ }^{5}$ Prof. do Depto. de Zootecnia da FZEA/USP, 13635-900, Pirassununga, SP. E.mail: vrherlin@usp.br

${ }^{6}$ Pesquisador da EMBRAPA Cerrados, 73301-970, Planaltina-DF. E.mail: allan@cpac.embrapa.br
} 
gem. Muitos solos são intemperizados e apresentam baixos teores de fósforo disponível (P). $\mathrm{O}$ fornecimento desse nutriente é importante, principalmente na fase inicial de estabelecimento da pastagem, pois proporciona condições favoráveis ao desenvolvimento de raízes e ao perfilhamento (Werner, 1986; Lobato et al., 1994).

No trabalho de Monteiro \& Werner (1977), a adubação fosfatada aumentou significativamente as produções de matéria seca (MS) e de proteína bruta (PB) na fase de estabelecimento do capim-colonião. Nesse mesmo trabalho, com o capim estabelecido, a adubação nitrogenada aumentou a produção de MS, o teor e a produção de $\mathrm{PB}$ da forragem.

$\mathrm{O}$ nitrogênio (N) é o nutriente mais influente para a produção de MS em pastagens já estabelecidas (Monteiro, 1995). O aumento da produção de forragem com a aplicação de $\mathrm{N}$ ocorre de forma linear e crescente (Vicente-Chandler, 1974; Gomide, 1989). O potencial de resposta das gramíneas forrageiras à adubação nitrogenada é um aspecto importante na escolha de cultivares para sistemas intensivos. Deve-se dirigir o manejo para o aproveitamento racional da forragem produzida, adequando a freqüência, a intensidade e a época de pastejo aos ganhos por animal e por área, evitando perdas no valor nutritivo da forragem. Todavia, é fundamental o balanço entre os nutrientes no solo, o qual pode interferir nos benefícios da adubação.

Gomide (1989) alertou que as relações inadequadas dos nutrientes, ou desequilíbrio dos minerais no solo podem interferir de maneira prejudicial na nutrição mineral das plantas, e conseqüentemente, limitar a produção de forragem. Como exemplo, podem ser mencionadas, entre outras, as relações do N com o P, com o enxofre e com o potássio (K) (Monteiro, 1995). Houve interação positiva entre N e K na produção de forragem do cv. Colonião adubado com $0,75,150$ e $250 \mathrm{~kg} / \mathrm{ha}$ de $\mathrm{N}$ e 0 e $100 \mathrm{~kg} / \mathrm{ha}$ de $\mathrm{K}_{2} \mathrm{O}$, evidenciando as afirmações de Monteiro et al. (1980).

As perdas de MS nas pastagens podem representar o resultado do acúmulo de material seco advindo das partes vegetais senescidas naturalmente, além do material verde, correspondente a perfilhos vivos, ou parte deles, quebrados ou tombados, devido à ação direta dos animais na pastagem. As perdas por aumento do pisoteio reduzem o aproveitamento da MS produzida (Maraschin, 1993; Nabinger, 1997).

Este trabalho foi conduzido com objetivo de avaliar os efeitos da adubação com NPK sobre os componentes da produção e das perdas de MS em pastagens dos cvs. Tanzânia e Mombaça de Panicum maximum Jacq.

\section{Material e Métodos}

O experimento foi conduzido de novembro de 1999 a abril de 2000, em área experimental de 2 ha, em pastagens estabelecidas há dois anos, localizada no Setor de Forragicultura da FCAV-Unesp, em Jaboticabal-SP $\left(21^{\circ} 15^{\prime} 22^{\prime \prime}\right.$ de latitude S, $48^{\circ} 18^{\prime} 58^{\prime \prime}$ de longitude $\mathrm{W}$ e $595 \mathrm{~m}$ de altitude). As avaliações foram realizadas em uma área de aproximadamente $2300 \mathrm{~m}^{2}$, dividida em 24 parcelas de $96 \mathrm{~m}^{2}$ cada uma, pastejadas simultaneamente, sendo metade delas estabelecidas com o cv. Tanzânia e metade com o cv. Mombaça de Panicum maximum Jacq. O restante da área estava constituído de piquetes de reserva (Brachiaria decumbens Stapf cv. Basilisk e Panicum maximum Jacq. cv. Aruana) e uma área de descanso com água e sal mineral para os animais.

$\mathrm{O}$ delineamento experimental adotado foi o de blocos completos casualizados para estudar o esquema fatorial 2 (cultivares) x 4 (doses de NPK), com três repetições. O solo da área foi classificado como Latossolo Vermelho.

As adubações variaram conforme a dose de NPK "padrão" adotada. A dose "padrão" foi calculada em relação às concentrações de 1,20; 0,08 e 1,20\% de N, $\mathrm{PeK}$ naMS da parte aérea, respectivamente, multiplicada pela produção de MS estimada de $12.000 \mathrm{~kg} / \mathrm{ha}$. A dose "padrão" de adubo foi reduzida em 30\% ("menos $30 "$ ), aumentada em $30 \%$ ("mais 30") e 60\% ("mais $60 ")$ para obtenção dos outros tratamentos. As doses de NPK ( $\mathrm{kg} / \mathrm{ha}$ ) aplicadas em cada tratamento estão relacionadas a seguir: "menos 30": 101,5 de N, $15,1 \mathrm{de}$ $\mathrm{P}_{2} \mathrm{O}_{5}$ e 126,0 de $\mathrm{K}_{2} \mathrm{O}$; "padrão": 145,0 de N, 21,6 de $\mathrm{P}_{2} \mathrm{O}_{5}$ e 180,0 de $\mathrm{K}_{2} \mathrm{O}$; "mais 30": 188,5 de N, 28,0 de $\mathrm{P}_{2} \mathrm{O}_{5}$ e 234,0 de $\mathrm{K}_{2} \mathrm{O}$; e "mais 60": 232,0 de N, 34,5 de $\mathrm{P}_{2} \mathrm{O}_{5}$ e 288,0 de $\mathrm{K}_{2} \mathrm{O}$. Os adubos utilizados foram o sulfato de amônio, o superfosfato simples e o cloreto de potássio.

Nas análises químicas, realizadas em outubro de 1999, em amostras de solo retiradas antes da adubação, à profundidade de $0 \mathrm{a} 20 \mathrm{~cm}$, foram obtidos os seguintes resultados médios: $\mathrm{pH} \mathrm{em} \mathrm{CaCl}_{2}=4,8 ; \mathrm{MO}=$ $21 \mathrm{~g} / \mathrm{dm}^{3} ;$ Presina $=4,2 \mathrm{mg} / \mathrm{dm}^{3} ; \mathrm{K}=4,7 \mathrm{mmol}_{\mathrm{c}} / \mathrm{dm}^{3}$; $\mathrm{Ca}=15,2 \mathrm{mmol}_{\mathrm{c}} / \mathrm{dm}^{3} ; \mathrm{Mg}=9,25 \mathrm{mmol}_{\mathrm{c}} / \mathrm{dm}^{3}$; $\mathrm{H}+\mathrm{Al}=28,7 \mathrm{mmol}_{\mathrm{c}} / \mathrm{dm}^{3} ; \mathrm{S}=29,2 \mathrm{mmol}_{\mathrm{c}} / \mathrm{dm}^{3}$; $\mathrm{T}=57,9 \mathrm{mmol}_{\mathrm{c}} / \mathrm{dm}^{3} ; \mathrm{e} \mathrm{V}=50 \%$.

Em novembro de 1999 foi realizado um corte de uniformização com roçadeira tratorizada, a $30 \mathrm{~cm} \mathrm{de}$ altura, sendo o material cortado retirado da área. Logo após, realizou-se a aplicação de 1,0 t/ha de

R. Bras. Zootec., v.31, n.3, p.1333-1342, 2002 (suplemento) 
calcário dolomítico $(\mathrm{PRNT}=91,5 \%)$, do adubo fosfatado e do potássico, além de $1 / 3$ do adubo nitrogenado, referentes às doses dos tratamentos.

Em janeiro de 2000, o primeiro pastejo foi realizado, e os dados não foram considerados, pelo longo período de descanso ( $>60$ dias). O pastejo foi efetuado por 3-4 novilhas e vacas secas da raça Holandesa, pesando em média $531 \mathrm{~kg}$, reunidas em dois grupos distintos para ocuparem os piquetes durante o dia e a noite, separadamente, visando a aceleração do rebaixamento das plantas.

Após esse primeiro pastejo, foi aplicada a dose correspondente ao segundo terço (1/3 do total) do adubo nitrogenado. O restante foi distribuído após o segundo pastejo, realizado no mês de fevereiro de 2000. Mais dois pastejos foram realizados, nos meses de março e abril de 2000.

A precipitação e as temperaturas médias mensais, de novembro de 1999 a abril de 2000, encontram-se na Figura 1. Esses dados foram fornecidos pela estação de Agrometeorologia do Departamento de Ciências Exatas da FCAV-UNESP, situada a $300 \mathrm{~m}$ da área experimental.

Omanejo da pastagem foi conduzido considerando-se 28 dias de descanso e a altura média do resíduo de $30 \mathrm{~cm}$, em relação à superfície do solo, como os fatores determinantes dos momentos de colocação e de retirada dos animais nos piquetes, respectivamente (Shaw et al., 1976).

Antes e após cada pastejo, a altura média das plantas foi determinada medindo-se em 10 touceiras por parcela, ao acaso, a distância do solo à curvatura da última folha completamente expandida, antes do pastejo, e a altura da extremidade das folhas pastejadas

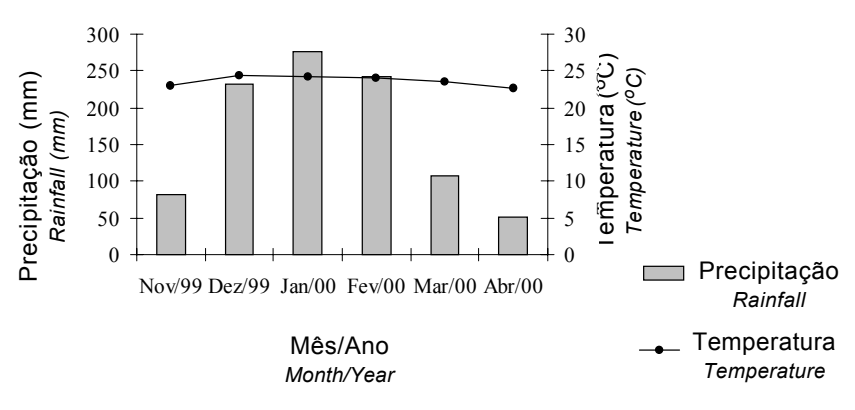

Figura 1 - Precipitação pluvial e temperatura média registradas durante o período de novembro de 1999 a abril de 2000.

Figure 1 - Rainfall and mean temperatures from November 1999 to April 2000.

R. Bras. Zootec., v.31, n.3, p.1333-1342, 2002 (suplemento) em relação ao solo, após o pastejo. A retirada de amostras foi realizada antes e após o pastejo, utilizando-se um quadrado de ferro de $0,5 \mathrm{~m}$ de lado, escolhendo-se ao acaso uma touceira para ser cortada no nível do solo. As amostras, devidamente identificadas, foram levadas ao laboratório para processamento. A seguir, procedeu-se à separação e à pesagem do material verde e do material senescido.

Os perfilhos verdes foram contados, e duas subamostras retiradas; uma delas, para a separação de lâminas foliares e de colmos (colmos + bainhas); a outra, para a determinação do teor de matéria seca dos perfilhos. Os teores de matéria seca dessas amostras foram determinados de acordo com a metodologia descrita por Silva (1998). A partir desses valores, estimaram-se a MSV em pré-pastejo e a MSV residual por hectare, os percentuais de lâminas foliares e de colmos, e a relação lâmina foliar/colmo, com base na MSV. A taxa de acúmulo diário de MSV foi estimada subtraindo-se da MSV em pré-pastejo a MSV residual do ciclo anterior, dividindo-se o resultado pelos dias de intervalo entre pastejos. O peso médio de perfilho foi determinado dividindo-se a MSV pelo número de perfilhos verdes obtidos na amostra. A densidade média de MSV $(\mathrm{kg} / \mathrm{ha} / \mathrm{cm}$ de altura) foi calculada dividindo-se a MSV pré-pastejo pela altura média das plantas.

O material senescido foi pesado e levado a estufa de ventilação forçada de ar a $60^{\circ} \mathrm{C}$ por 72 horas para a determinação do percentual de MS. Assim, foram estimados a quantidade de MS senescida e o seu percentual em relação à MS total da parte aérea $(\mathrm{MST}=\mathrm{MSV}+\mathrm{MS}$ senescida $)$.

As perdas de MSV por pisoteio foram quantificadas marcando-se uma área de $2 \times 1 \mathrm{~m}$ em cada parcela, colhendo-se toda a massa verde tombada (presa à planta ou não), imediatamente após a saída dos animais do piquete. O material, após identificação, foi levado ao laboratório para ser pesado. Foram retiradas duas subamostras representativas: uma delas para a quantificação da proporção de lâminas foliares e de colmos e a relação lâmina foliar/ colmo; a outra para determinar o teor de MS.

Os animais utilizados foram pesados em cada ciclo de pastejo, e, ao término do experimento, foi calculado o número de unidades animais (UA) por unidade de tempo necessário para rebaixar a forragem até a altura desejável de resíduo. O valor obtido foi ajustado para 30 dias ( 28 dias de descanso mais dois dias de ocupação, em média). 
Os dados foram analisados pelo SAS (SAS, 1996). Os efeitos de cultivar (C), de adubação (A) e da interação $\mathrm{C}$ x A foram analisados nas parcelas, enquanto os efeitos de época de avaliação (E) e as suas interações foram analisados nas subparcelas, sendo as médias dos cultivares comparadas pelo teste de Tukey a $5 \%$ de probabilidade. O efeito da adubação foi estudado usando o desdobramento dos graus de liberdade em efeito linear, quadrático e cúbico.

\section{Resultados e Discussão}

A MSV pré-pastejo média, em função das doses de NPK, diferiu $(\mathrm{p}<0,01)$ entre cultivares, e o cv. Mombaça apresentou valores mais elevados do que o cv. Tanzânia (Tabela 1).

A MSV em pré-pastejo nem sempre traduz completamente o potencial de produção do cultivar, pois deve ser considerada a MS residual anterior. Nesse sentido, a taxa de acúmulo diário de MSV foi maior $(\mathrm{p}<0,05)$ no cv. Mombaça do que no cv. Tanzânia e aumentou linearmente com as doses de NPK aplicadas para ambos os cultivares (Tabela 1). Esses resultados corroboram os obtidos por Santos et al. (1999), que citaram produções do cv. Mombaça cerca de 30 \% maiores $(\mathrm{p}<0,05)$ do que as do cv. Tanzânia.

A adubação com NPK aumentou a MSV em prépastejo e a taxa de acúmulo diário de MSV. A taxa de acúmulo diário de MSV média dos cultivares foi de $115 \mathrm{~kg} / \mathrm{ha} / \mathrm{dia}$ nas doses "menos 30" e "padrão", respectivamente, alcançando $186 \mathrm{~kg} / \mathrm{ha} /$ dia na dose "mais 60". Pieterse et al. (1997) obtiveram respostas lineares e crescentes na produção de MS dos cvs. Mutale, Petrie e Gatton de Panicum maximum, quando aumentaram as doses de $\mathrm{N}$ de 0 para $320 \mathrm{~kg} / \mathrm{ha}$.

As taxas de acúmulo diário de MSV foram decrescentes $(p<0,05)$ ao longo do período experimental, sendo de 183, 101 e $93 \mathrm{~kg} / \mathrm{ha} / \mathrm{dia}$ de MSV no cv. Tanzânia e de 186, 166 e 149 kg/ha/dia de MSV no cv. Mombaça, referentes aos meses de fevereiro, março e abril, respectivamente, o que pode ser atribuído às condições climáticas menos favoráveis em relação às do início da estação de crescimento.

A MSV média dos dois cultivares em pré-pastejo diminuiu $(\mathrm{p}<0,01)$ da primeira para a última avaliação, sendo de 9498,8156 e $5676 \mathrm{~kg} / \mathrm{ha}$, relativas às épocas de fevereiro, março e abril, respectivamente, devido, provavelmente, ao regime das precipitações registradas durante o período experimental (Figura 1).

O percentual de lâminas foliares da MSV do cv.
Mombaça (54,3\%) foi maior que o do cv. Tanzânia $(51,1 \%)(\mathrm{p}<0,05)$ (Tabela 1). Jank (1995) relatou que a porcentagem de lâminas foliares do cv. Mombaça foi de $82 \%$, valor esse que foi ligeiramente superior ao do cv. Tanzânia, com $80 \%$, enquanto a produção de MS de lâminas foliares foi de 33000 e $26000 \mathrm{~kg} / \mathrm{ha}$, respectivamente. Os percentuais de lâminas foliares obtidos no presente trabalho foram inferiores aos citados por Jank (1995), provavelmente em decorrência da aplicação de maiores doses de fertilizantes, $o$ que, segundo Forni et al. (2000), resulta em maior desenvolvimento de colmos para sustentação de maiores produções de lâminas foliares.

Cecato et al. (2000) encontraram percentuais de lâminas foliares na MSV dos cv. Tanzânia e Mombaça de 81 e $78 \%$ e 89 e $85 \%$, respectivamente para os cortes nas alturas de 20 e $40 \mathrm{~cm}$, sendo os resultados obtidos no presente trabalho inferiores, o que pode ser atribuído, entre outros fatores, à amostragem das plantas no nível do solo. Além disso, o primeiro ciclo de pastejo ocorreu 55-65 dias após o corte de uniformização, o que resultou em acúmulo de quantidades excessivas de colmos, provocando outro tipo de resposta.

A densidade de perfilhos não variou com a adubação e nem entre cultivares. Assim, o aumento da MSV em pré-pastejo com a adubação foi conseqüência do aumento do peso médio dos perfilhos (Tabela 1), corroborando os resultados obtidos por Herling (1995), quando adubou o capim-colonião com doses variando de 0 a $320 \mathrm{~kg} / \mathrm{ha}$ de $\mathrm{N}$.

$\mathrm{O}$ cv. Mombaça apresentou produção de MSV superior à do cv. Tanzânia, devido ao maior peso dos perfilhos, pois ambos os cultivares mostraram densidades semelhantes.

No entanto, a densidade de perfilhos, antes do pastejo, diminuiu $(\mathrm{p}<0,05)$ com o avanço da época de avaliação, sendo as médias dos cultivares e das doses de NPK de 706, 671 e 523 perfilhos $/ \mathrm{m}^{2}$, nos meses de fevereiro, março e abril, respectivamente. O peso dos perfilhos foi influenciado $(\mathrm{p}<0,01)$ pelo cultivar, pela adubação e também pela época de avaliação $(1,47$; 1,23 e 1,08 g de MS/perfilho nos meses de fevereiro, março e abril, respectivamente), de modo semelhante ao ocorrido com a população de perfilhos.

A altura média das plantas, antes do pastejo aumentou $(p<0,01)$ com a elevação das doses de NPK aplicadas, sendo a altura do cv. Mombaça $(77,6 \mathrm{~cm})$ superior $(p<0,05)$ à do cv. Tanzânia $(63,9 \mathrm{~cm})$. A densidade de MSV aumentou $(\mathrm{p}<0,05)$ com a adubação, sendo de 105, 102, 107 e 121 kg/ha/cm de MSV

R. Bras. Zootec., v.31, n.3, p.1333-1342, 2002 (suplemento) 
Tabela 1 - Componentes da produção de matéria seca (MS) antes do pastejo e taxa de acúmulo diário de matéria seca verde (MSV) em pastagens dos cvs. Tanzânia e Mombaça de Panicum maximum Jacq. adubadas com quatro doses de NPK

Table 1 - Components of dry matter (DM) production and green dry matter (GDM) accumulation rate in pastures of the cultivars Tanzania and Mombaça of Panicum maximum Jacq. fertilized with four doses of NPK

\begin{tabular}{|c|c|c|c|c|c|c|}
\hline \multirow{3}{*}{$\begin{array}{l}\text { Cultivar } \\
\text { Cultivar }\end{array}$} & \multicolumn{6}{|c|}{$\begin{array}{c}\text { Doses de } \mathrm{NPK}^{1} \\
\text { NPK doses }\end{array}$} \\
\hline & Menos30 & Padrão & Mais30 & Mais60 & Média & $e f^{2}$ e valor de $p$ \\
\hline & Minus30 & Standard & Plus30 & Plus60 & Mean & $E f^{2}$ and $p$ value \\
\hline & \multicolumn{6}{|c|}{ MSV (kg/ha) } \\
\hline & \multicolumn{6}{|c|}{$G D M(k g / h a)$} \\
\hline Mombaça & 8744 & 8198 & 7937 & 11854 & $9183 \mathrm{~A}^{3}$ & \\
\hline Tanzânia & 4334 & 6196 & 7446 & 7230 & $6275 \mathrm{~B}$ & \\
\hline \multirow[t]{3}{*}{ Média (Mean) } & 6539 & 7197 & 7692 & 9678 & $\mathrm{CV}^{4}=31,9 \%$ & ef $L, p<0,01$ \\
\hline & \multicolumn{6}{|c|}{ \% lâminas foliares da MSV } \\
\hline & \multicolumn{6}{|c|}{ Leaf blade \% in the GDM } \\
\hline Mombaça & 54,3 & 55,3 & 51,6 & 56,1 & $54,3 \mathrm{~A}$ & \\
\hline Tanzânia & 52,4 & 54,9 & 49,2 & 48,0 & $51,1 \mathrm{~B}$ & \\
\hline \multirow[t]{3}{*}{ Média (Mean) } & 53,4 & 55,1 & 50,4 & 52,1 & $\mathrm{CV}=9,2 \%$ & efC, $\mathrm{p}<0,05$ \\
\hline & \multicolumn{6}{|c|}{$\%$ colmos da MSV } \\
\hline & \multicolumn{6}{|c|}{ Stem \% in the GDM } \\
\hline Mombaça & 45,6 & 44,6 & 48,3 & 43,8 & $45,6 \mathrm{~B}$ & \\
\hline Tanzânia & 47,5 & 45,0 & 50,7 & 51,9 & $48,8 \mathrm{~A}$ & \\
\hline \multirow[t]{3}{*}{ Média (Mean) } & 46,5 & 44,8 & 49,5 & 47,8 & $\mathrm{CV}=9,2 \%$ & efC, $\mathrm{p}<0,05$ \\
\hline & \multicolumn{6}{|c|}{ Densidade de perfilhos $\left(\mathrm{n}^{\circ} / \mathrm{m}^{2}\right)$} \\
\hline & \multicolumn{6}{|c|}{ Tiller density $\left(n^{o} / m^{2}\right)$} \\
\hline Mombaça & 639 & 611 & 554 & 660 & 616 & \\
\hline Tanzânia & 628 & 696 & 704 & 585 & 653 & \\
\hline \multirow[t]{3}{*}{ Média (Mean) } & 634 & 656 & 629 & 623 & $\mathrm{CV}=17,0 \%$ & $\mathrm{~ns}$ \\
\hline & \multirow{2}{*}{\multicolumn{6}{|c|}{ Peso de perfilho $(\mathrm{g})$}} \\
\hline & & \multicolumn{5}{|c|}{ Tiller weight $(g)$} \\
\hline Mombaça & 1,3 & 1,4 & 1,4 & 1,7 & $1,5 \mathrm{~A}$ & \\
\hline Tanzânia & 0,6 & 0,8 & 1,0 & 1,4 & $1,0 \mathrm{~B}$ & \\
\hline \multirow[t]{3}{*}{ Média (Mean) } & 1,0 & 1,1 & 1,2 & 1,6 & $\mathrm{CV}=26,0 \%$ & ef $L, p<0,01$ \\
\hline & \multicolumn{6}{|c|}{ Altura média das plantas $(\mathrm{cm})$} \\
\hline & \multicolumn{6}{|c|}{ Average plants height $(\mathrm{cm})$} \\
\hline Mombaça & 69,8 & 78,7 & 78,7 & 82,1 & $77,3 \mathrm{~A}$ & \\
\hline Tanzânia & 53,3 & 62,3 & 65,9 & 73,9 & $63,9 \mathrm{~B}$ & \\
\hline \multirow[t]{3}{*}{ Média (Mean) } & 61,5 & 70,5 & 72,3 & 78,0 & $C V=13,3 \%$ & ef $\mathrm{L}, \mathrm{p}<0,01$ \\
\hline & \multicolumn{6}{|c|}{ MS senescida $(\mathrm{kg} / \mathrm{ha})$} \\
\hline & \multicolumn{6}{|c|}{ Senesced DM $(\mathrm{kg} / \mathrm{ha})$} \\
\hline Mombaça & 2850 & 2520 & 4258 & 3354 & 3246 & \\
\hline Tanzânia & 3376 & 2409 & 3797 & 3114 & 3174 & \\
\hline Média (Mean) & 3113 & 2464 & 4028 & 3234 & $\mathrm{CV}=25,0 \%$ & efC, $p=0,01$ \\
\hline & & & $\% \mathrm{MS}$ ser & da MST & & \\
\hline & & & $\%$ senesc & in $T D M^{5}$ & & \\
\hline Mombaça & 31,5 & 24,2 & 36,2 & 28,0 & $30,0 \mathrm{~B}$ & \\
\hline Tanzânia & 41,9 & 31,3 & 40,7 & 28,9 & $35,7 \mathrm{~A}$ & \\
\hline Média (Mean) & 36,7 & 27,8 & 38,5 & 28,4 & $\mathrm{CV}=17,5 \%$ & efC, $p=0,01$ \\
\hline & & & le acúmulo d & $\operatorname{MSV}(\mathrm{k}$ & lia) & \\
\hline & & & GDM accum & rates $(\mathrm{kg})$ & & \\
\hline Mombaça & 144 & 128 & 191 & 205 & $167 \mathrm{~A}$ & \\
\hline Tanzânia & 87 & 103 & 144 & 167 & $125 \mathrm{~B}$ & \\
\hline Média (Mean) & 115 & 115 & 168 & 186 & $\mathrm{CV}=49,5 \%$ & ef $L, p<0,05$ \\
\hline
\end{tabular}

${ }^{1}$ A dose de NPK "Padrão" corresponde a 145, 21,6 e 180 kg/ha de N, P2O5 e K2O, respectivamente. Menos30, Mais30 e Mais60 correspondem ao decréscimo em $30 \%$ e ao acréscimo em 30 e $60 \%$ da "Padrão", respectivamente ("Standard" correponded to the aplication of 145, 21.6 and $180 \mathrm{~kg} / \mathrm{ha}$ of N, P2O5 and K2O, respectively. Minus30, Plus 30 and Plus60 corresponded to the reduction of $30 \%$ and increase of 30 and $60 \%$ in relation to the "Standard" dose, respectively).

2 Efeito da adubação: ef $L=$ efeito linear, ef $Q=$ efeito quadrático, ef $C=$ efeito cúbico e ns=não significativo (Effect of fertilization: ef $L=$ linear effect, ef $Q=q u a d r a t i c$ effect, ef $C=$ cubic effect and $n s=$ non significant).

${ }^{3}$ Letras diferentes na mesma coluna diferem $(p<0,05)$ pelo Teste de Tukey (Different letters in the same column differ $(p<0.05)$ by the test of Tukey).

${ }^{4} \mathrm{CV}=$ coeficiente de variação ( $\mathrm{CV}=$ coefficient of variation).

${ }^{5}$ MST $($ MS total da parte aérea $)=$ MSV + MS senescida $(T D M($ total shoot $D M)=G D M+$ senesced $D M)$. 
nas doses de adubação "menos 30", "padrão", "mais 30 " e "mais 60", respectivamente. De modo geral, o cv. Mombaça apresentou uma densidade de MSV superior $(\mathrm{p}<0,05)(119 \mathrm{~kg} / \mathrm{ha} / \mathrm{cm}$ de MSV $)$ à do cv. Tanzânia ( $99 \mathrm{~kg} / \mathrm{ha} / \mathrm{cm}$ de MSV).

Houve interação $(p<0,05)$ adubação $x$ época na MS senescida, sendo de 2402, 3474 e $3464 \mathrm{~kg} / \mathrm{ha}$ de MS na dose de NPK "menos 30"; 2839, 1780 e 2774 kg/ ha de MS na "padrão"; 4526, 4250 e 3307 kg/ha de MS na "mais 30"; e 2108, 3243 e 4351 kg/ha de MS na "mais $60 "$, referente aos meses de fevereiro, março e abril, respectivamente. Mazzanti \& Lemaire (1994) não observaram efeito definido da adubação nitrogenada e da época do ano sobre a dinâmica da taxa de senescência de festuca (Festuca arundinacea Schreb.), sob lotação contínua e carga variável, durante dois anos de avaliação. Entretanto, verificaram efeitos mais marcantes do cultivar e da manutenção de um índice de área foliar (IAF) igual a três, em comparação com IAF igual a dois, o que contribuiu para o aumento da taxa de senescência de 22,7 para $30,4 \mathrm{~kg} /$ ha/dia de matéria orgânica, respectivamente.

Houve efeitos significativos $(\mathrm{p}<0,01)$ do cultivar, da adubação, da época e da interação adubação $x$ época $(\mathrm{p}<0,05)$ no percentual de MS senescida da MST pré-pastejo, aumentando à medida que avançou a estação chuvosa do ano. Nos meses de fevereiro, março e abril, o percentual de MS senescida da MST em pré-pastejo foi de 24,35 e $50 \%$ na dose "menos $30 " ; 24,18$ e 40 \% na "padrão"; 31, 32 e $51 \%$ na "mais 30"; e 15, 24 e $45 \%$ na "mais 60", respectivamente. Euclides et al. (1999) observaram que a participação de MS senescida na pastagem é estacional, sendo bem superior na época seca do ano. Os valores mais elevados de MS senescida antes do pastejo foram observados nos tratamentos "menos 30" e "mais 30". Por outro lado, os valores encontrados nos tratamentos "padrão" e "mais 60" foram semelhantes, mas não se refletiram na quantidade de MS senescida devido à produção de MS e à estrutura do pasto.

O cv. Tanzânia apresentou maior percentual de MS senescida em relação a MST, embora os valores obtidos em ambos os cultivares no tratamento "mais $60 "$ tenham se aproximado. Antes do pastejo, o percentual de MS senescida no cv. Tanzânia (36\%) foi maior do que no cv. Mombaça (30\%), provavelmente em decorrência do auto-sombreamento causado pela disposição mais decumbente das folhas neste cultivar quando comparado ao cv. Mombaça.

A MSV residual média de todas as doses de NPK do cv. Mombaça $(5279 \mathrm{~kg} / \mathrm{ha})$ foi maior $(\mathrm{p}<0,05)$ do que a do cv. Tanzânia (3808 kg/ha), o que influenciou os resultados da MSV em pré-pastejo, pois a rebrota é originada da MSV residual (Tabela 2).

O resíduo na faixa de 3000-3500 kg/ha de MSV foi conseguido no cv. Tanzânia até a dose de adubação "mais 30" (Tabela 2). Considerou-se esse aspecto como uma facilidade maior de manejo do cv. Tanzânia, em comparação com o cv. Mombaça. Na dose de adubação "mais 60", a MSV residual de ambos os cultivares aumentou e foi semelhante. Mesmo que os cultivares sejam manejados com intervalos de pastejo reduzidos (28 dias), adubações com altas doses de NPK resultaram em maiores MSV residuais, de maneira semelhante ao ocorrido com a altura média das plantas.

Teixeira et al. (1999), trabalhando com o cv. Tobiatã, obtiveram resíduo médio de $5554 \mathrm{~kg} / \mathrm{ha}$ de MSV. Contudo, houve variação com o avanço da estação de pastejo, de 1491 a $8089 \mathrm{~kg} / \mathrm{ha}$ de MSV, do primeiro ao quarto ciclo. Por outro lado, no presente trabalho, a MSV residual não foi influenciada pela época, sendo de 4810, 4750 e $4048 \mathrm{~kg} / \mathrm{ha}$ para os ciclos de pastejo efetuados em fevereiro, março e abril, respectivamente.

A interação cultivar $x$ adubação foi significativa $(p<0,05)$ no percentual de lâminas foliares da MSV residual. Essa interação decorreu provavelmente do acúmulo de colmos nos piquetes mais adubados do cv. Mombaça, o que dificultou o manejo uniforme das pastagens. Entretanto, foram mantidas quantidades razoáveis de lâminas foliares verdes em todos os tratamentos. Esses resultados demonstraram que a adubação com doses de NPK acima da dose "mais 30" reduziu o percentual de lâminas foliares no resíduo a patamares inferiores a $20 \%$ da MSV no cv. Mombaça (Tabela 2), semelhantemente aos valores encontrados por Teixeira et al. (1999) para o cv. Tobiatã.

Comparando-se os dados das Tabelas 1 e 2, percebe-se que, da forragem em pré-pastejo para a residual, houve redução na densidade de perfilhos, provavelmente devido ao pisoteio, ao arranquio ou ao consumo. Os valores da participação (\%) de lâminas foliares foram sempre inferiores no resíduo em relação ao pré-pastejo, indicando a ocorrência de seleção desse componente pelos animais (Euclides et al., 1999).

Mesmo mantendo a altura de resíduo próxima daquela pré-determinada, observou-se que a MSV variou de 2955 a $6185 \mathrm{~kg} / \mathrm{ha}$, indicando que a altura de resíduo não é um bom parâmetro de manejo, porque

R. Bras. Zootec., v.31, n.3, p.1333-1342, 2002 (suplemento) 
os componentes morfológicos são alterados em função do cultivar e da adubação utilizada.

A MS senescida não diferiu entre os cultivares, tanto em pré- (Tabela 1) como em pós-pastejo (Tabela 2). O valor médio da MS senescida antes e depois do pastejo, para os dois cultivares, foi de $3108 \mathrm{~kg} / \mathrm{ha}$. Esse resultado foi superior ao obtido por Sisti et al.
(1999), que encontraram MS senescida de $2107 \mathrm{~kg} / \mathrm{ha}$ no cv. Mombaça com período de descanso de 28 dias.

Entretanto, o percentual de MS senescida da MS residual (MSR $=\mathrm{MSV}+\mathrm{MS}$ senescida) foi maior no $\mathrm{cv}$. Tanzânia (44,1 \%) do que no cv. Mombaça (37,7 \%) (Tabela2), provavelmente em conseqüência do percentual de MS senescida da MST em pré-pastejo (Tabela 1).

Tabela 2 - Componentes da matéria seca (MS) residual dos cvs. Tanzânia e Mombaça de Panicum maximum Jacq. adubados com quatro doses de NPK

Table 2 - Components of the residual dry matter (RDM) of Tanzania and Mombaça pastures fertilized with four doses of NPK

\begin{tabular}{|c|c|c|c|c|c|c|}
\hline \multirow{3}{*}{$\begin{array}{l}\text { Cultivar } \\
\text { Cultivar }\end{array}$} & \multicolumn{6}{|c|}{$\begin{array}{c}\text { Doses de } \mathrm{NPK}^{1} \\
N P K \text { doses }\end{array}$} \\
\hline & Menos 30 & Padrão & Mais30 & Mais60 & Média & $e f^{2}$ e valor de $p$ \\
\hline & Minus30 & Standard & Plus30 & Plus60 & Mean & $e f^{2}$ and $p$ value \\
\hline & \multicolumn{6}{|c|}{$\mathrm{MSV}^{5}$ residual $(\mathrm{kg} / \mathrm{ha})$} \\
\hline & \multicolumn{6}{|c|}{ Residual GDM ${ }^{5}(\mathrm{~kg} / \mathrm{ha})$} \\
\hline Mombaça & 4404 & 6085 & 4520 & 6109 & $5279 A^{3}$ & \\
\hline Tanzânia & 2955 & 3300 & 3402 & 5574 & 3808B & \\
\hline \multirow[t]{3}{*}{ Média (Mean) } & 3679 & 4658 & 3961 & 5841 & $\mathrm{CV}^{4}=33,5 \%$ & ef $L, p<0,01$ \\
\hline & \multicolumn{6}{|c|}{ \% lâminas da MSV residual } \\
\hline & \multicolumn{6}{|c|}{ Leaf blade $\%$ in the residual GDM } \\
\hline Mombaça & 34,36 & 32,24 & 21,75 & 17,86 & 26,22 & ef $L, p<0,01$ \\
\hline Tanzânia & 31,18 & 23,25 & 29,74 & 25,89 & 27,41 & ns \\
\hline \multirow{3}{*}{ Média (Mean) } & 32,86 & 27,18 & 25,74 & 21,88 & $\mathrm{CV}=19,5 \%$ & \\
\hline & \multicolumn{6}{|c|}{$\%$ colmos da MSV residual } \\
\hline & \multicolumn{6}{|c|}{ Stem \% in the residual GDM } \\
\hline Mombaça & 65,63 & 67,75 & 78,25 & 82,33 & 73,77 & ef $L, p<0,01$ \\
\hline Tanzânia & 68,81 & 76,74 & 70,25 & 74,10 & 72,58 & ns \\
\hline \multirow[t]{3}{*}{ Média (Mean) } & 67,13 & 72,81 & 74,25 & 78,11 & $\mathrm{CV}=7,1 \%$ & \\
\hline & \multirow{2}{*}{\multicolumn{6}{|c|}{$\begin{array}{l}\text { Densidade de perfilhos }\left(\mathrm{n}^{\mathrm{o}} / \mathrm{m}^{2}\right) \\
\text { Tiller density }\left(\mathrm{n}^{\circ} / \mathrm{m}^{2}\right)\end{array}$}} \\
\hline & & & & & & \\
\hline Mombaça & 576 & 559 & 597 & 528 & 565 & \\
\hline Tanzânia & 641 & 606 & 538 & 587 & 593 & \\
\hline \multirow[t]{3}{*}{ Média (Mean) } & 608 & 583 & 568 & 557 & $\mathrm{CV}=19,9 \%$ & $\mathrm{~ns}$ \\
\hline & \multicolumn{6}{|c|}{ Altura média das plantas $(\mathrm{cm})$} \\
\hline & \multicolumn{6}{|c|}{ Average plant height $(\mathrm{cm})$} \\
\hline Mombaça & 32,3 & 34,4 & 35,3 & 37,3 & $34,8 \mathrm{~A}$ & \\
\hline Tanzânia & 31,5 & 31,5 & 35,9 & 33,3 & $33,0 \mathrm{~B}$ & \\
\hline \multirow[t]{3}{*}{ Média (Mean) } & 31,9 & 32,9 & 35,6 & 35,3 & $\mathrm{CV}=5,2 \%$ & ef $L, p<0,01$ \\
\hline & \multicolumn{6}{|c|}{ MS senescida $(\mathrm{kg} / \mathrm{ha})$} \\
\hline & \multicolumn{6}{|c|}{ Senesced DM $(\mathrm{kg} / \mathrm{ha})$} \\
\hline Mombaça & 2496 & 4073 & 3240 & 2957 & 3166 & efQ, $p=0,05$ \\
\hline Tanzânia & 2766 & 2275 & 2460 & 3879 & 2845 & efQ, $p=0,05$ \\
\hline \multirow[t]{3}{*}{ Média (Mean) } & 2631 & 3121 & 2850 & 3418 & $\mathrm{CV}=22,9 \%$ & \\
\hline & \multirow{2}{*}{\multicolumn{6}{|c|}{$\begin{array}{l}\% \text { MS senescida da } \mathrm{MSR}^{6} \\
\% \text { senesced } D M \text { to } R D M^{6}\end{array}$}} \\
\hline & & & & & & \\
\hline Mombaça & 36,6 & 40,7 & 40,7 & 33,1 & $37,7 \mathrm{~B}$ & \\
\hline Tanzânia & 48,4 & 40,3 & 42,0 & 46,0 & $44,1 \mathrm{~A}$ & \\
\hline Média (Mean) & 42,5 & 40,5 & 41,4 & 39,2 & $\mathrm{CV}=16,2 \%$ & ns \\
\hline
\end{tabular}

${ }^{1}$ A doses de NPK "Padrão" corresponde a 145, 21,6 e $180 \mathrm{~kg} / \mathrm{ha}$ de $\mathrm{N}, \mathrm{P}_{2} \mathrm{O}_{5}$ e $\mathrm{K} 2 \mathrm{O}$, respectivamente. Menos 30 , Mais30 e Mais60 correspondem ao decréscimo em $30 \%$ e ao acréscimo em 30 e $60 \%$ da dose de NPK "Padrão", respectivamente "Standard" dose correponded to the aplication of $145,21.6$ and $180 \mathrm{~kg} / \mathrm{ha}$ of $\mathrm{N}, \mathrm{P}_{2} \mathrm{O}_{5}$ and $\mathrm{K}_{2} \mathrm{O}$, respectively. Minus 30 , Plus 30 and Plus 60 corresponded to the reduction of $30 \%$ and increase of 30 and $60 \%$ in relation to the "Standard" dose, respectively).

${ }^{2}$ Efeito da adubação: ef $\mathrm{L}=$ efeito linear, ef $\mathrm{Q}=$ efeito quadrático, ef $\mathrm{C}=$ efeito cúbico e $n s=$ não significativo (Effect of fertilization: ef $L=$ linear effect, ef $Q=q u a d r a t i c$ effect, ef $C=$ cubic effect and $n s=$ non significant).

${ }^{3}$ Letras diferentes na mesma coluna diferem $(p<0,05)$ pelo Teste de Tukey (Different letters in the same column differ $(p<0.05)$ by the test of Tukey).

${ }^{4} \mathrm{CV}=$ coeficiente de variação ( $C V=$ coefficient of variation).

${ }^{5} \mathrm{MSV}=\mathrm{MS}$ verde $(G D M=$ green $D M)$.

${ }^{6} \mathrm{MS}$ residual $(\mathrm{MSR})=\mathrm{MSV}+\mathrm{MS}$ senescida $($ Residual $D M(R D M)=G D M+$ senesced $D M)$.

\section{R. Bras. Zootec., v.31, n.3, p.1333-1342, 2002 (suplemento)}


Considerando a MS senescida em pré- (Tabela 1) e pós-pastejo (Tabela 2), notou-se que a localização da maior parte desse material ocorreu abaixo da altura de manejo utilizada, interferindo pouco na qualidade da forragem disponível.

$\mathrm{O}$ cv. Mombaça apresentou perdas de MSV por pisoteio mais elevadas $(644 \mathrm{~kg} / \mathrm{ha})(\mathrm{p}<0,05)$ do que o cv. Tanzânia (437 kg/ha) (Tabela 3). Contudo, os valores médios do percentual de MSV perdida por pisoteio em relação a MSV em pré-pastejo dos dois cultivares foram semelhantes $(p>0,05)$, com média de $7,7 \%$.

A adubação com doses mais elevadas de NPK aumentou a MSV perdida por pisoteio e seu percentual em relação à MST em pré-pastejo, aumento esse provavelmente relacionado à MSV em pré-pastejo. Na dose "mais 60", em ambos os cultivares, a MSV perdida por pisoteio esteve próxima ao resultado médio de $1179 \mathrm{~kg} / \mathrm{ha}$, obtido por Teixeira et al. (1999) no cv. Tobiatã entre os meses de novembro e maio.

Os resultados do percentual de MSV perdida por pisoteio em relação à MSV em pré-pastejo (2,0 a 14,5 $\%$ ) (Tabela 3) podem ser considerados normais se comparados com os 9,2 \% no cv. Tobiatã e cerca de $14 \%$ no cv. Tanzânia obtidos por Teixeira et al. (1999) e Rosseto (2000), respectivamente.

As maiores quantidades de MSV perdida por pisoteio obtidas nos piquetes do cv. Mombaça (Tabela 3) podem estar relacionadas com a maior MSV (30 $\%)$ e a altura mais elevada (20\%) das plantas (Tabela 1). Sem dúvida, as gramíneas forrageiras cespitosas eretas são mais vulneráveis ao amassamento e ao pisoteio (Humphreys, 1991).

A participação dos colmos nas perdas por pisoteio aumentou linearmente com a adubação $(\mathrm{p}<0,05)$. Na MSV do cv. Tanzânia perdida por pisoteio, observouse, de modo geral, maior percentual de colmos $(\mathrm{p}<0,05)$ do que na do cv. Mombaça.

A maior proporção de lâminas foliares perdidas no cv. Mombaça (Tabela 3) segue, em parte, as características da MSV em pré-pastejo (Tabela 1), apesar de não ter atingido valores médios tão altos.

Ao comparar as características (percentuais de lâminas foliares e de colmos) das perdas por pisoteio (Tabela 3) com a da MS pré-pastejo (Tabela 1), percebe-se que houve mais colmos nas perdas, devido ao tombamento de perfilhos parcialmente pastejados. Portanto, essa observação sugere que as perdas ocorreram ao longo do pastejo, mas foram mais elevadas no início do pastejo, com a densidade de MSV mais elevada, conforme o afirmado por Vallentine (1990).

A adubação e o cultivar influenciaram significativamente $(p<0,01)$ a taxa de lotação estimada nos piquetes. (Figura 2). O maior aporte de nutrientes advindo do aumento da adubação com NPK resultou em um aumento da taxa de lotação. Com base nesses resultados, pode-se inferir que existe a possibilidade do aumento da produção animal com a maior produção de forragem. Deve ser ressaltado que o desempenho animal não foi mensurado. Incrementos na taxa de lotação também foram verificados por Corrêa (2000) quando as doses de $\mathrm{N}$ foram elevadas de 200 para $300 \mathrm{~kg} / \mathrm{ha}$.

A taxa de lotação estimada do cv. Mombaça $(6,2 \mathrm{UA} / \mathrm{ha})$ foi $50 \%$ maior $(\mathrm{p}<0,05)$ do que a do cv. Tanzânia (4,0 UA/ha). Esses resultados estão relacionados com os da taxa de acúmulo diário de MSV, os quais foram mais altos no cv. Mombaça do que no cv. Tanzânia (Tabela 1). Cecato et al. (2000) destacaram o cv. Mombaça como um dos mais produtivos na época das "águas", dentre os seis cultivares e os dois acessos de Panicum maximum avaliados.

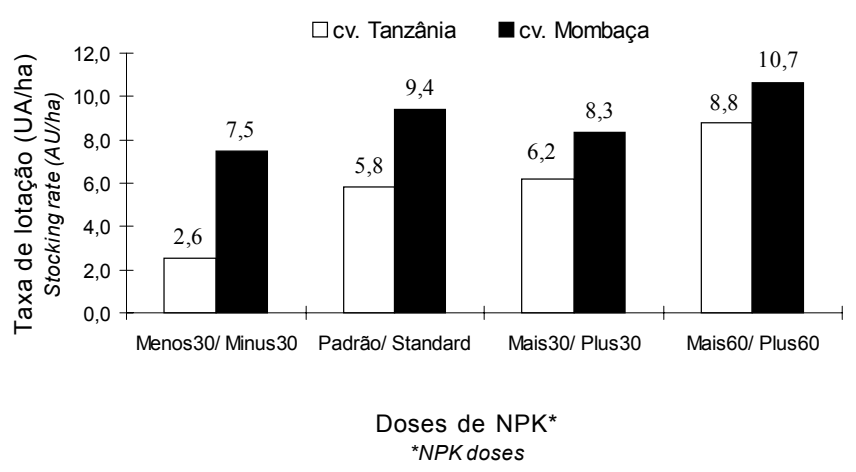

Figura 2 - Taxa de lotação estimada nos piquetes dos cvs. Tanzânia e Mombaça de Panicum maximum Jacq. adubados com quatro doses de NPK (Coeficiente de variação=25,0\%). * A dose "padrão" corresponde a 145, 21,6 e $180 \mathrm{~kg} / \mathrm{ha}$ de $\mathrm{N}, \mathrm{P}_{2} \mathrm{O}_{5}$ e $\mathrm{K}_{2} \mathrm{O}$, respectivamente. Menos30, Mais 30 e Mais60 correspondem ao decréscimo em $30 \%$, ao acréscimo em 30 e $60 \%$ da dose de NPK "padrão", respectivamente.

Figure 2 - Estimated stocking rate in the paddocks of the cvs. Tanzania and Mombaça, fertilised with four doses of NPK (Coefficient of variation $=25.0 \%$ ).

* The "standard" dose correponded to the aplication of $145,21.6$ and $180 \mathrm{~kg} / \mathrm{ha}$ of $\mathrm{N}, \mathrm{P}_{2} \mathrm{O}_{5}$ and $\mathrm{K}_{2} \mathrm{O}$, respectively. Minus30, Plus 30 and Plus60 corresponded to the reduction of $30 \%$, and increase of 30 and $60 \%$ in relation to the "standard" dose, respectively. 
Tabela 3 - Componentes da matéria seca verde (MSV) perdida por pisoteio em pastagens dos cvs. Tanzânia e Mombaça de Panicum maximum Jacq. adubadas com quatro doses de NPK

Table 3 - Green dry matter (GDM) losses by trampling and their components in the cvs. Tanzania and Mombaça pastures fertilized with four doses of NPK

\begin{tabular}{|c|c|c|c|c|c|c|}
\hline \multirow{3}{*}{$\begin{array}{l}\text { Cultivares } \\
\text { Cultivars }\end{array}$} & \multicolumn{6}{|c|}{$\begin{array}{c}\text { Doses de } \mathrm{NPK}^{1} \\
N P K \text { doses }\end{array}$} \\
\hline & Menos30 & Padrão & Mais30 & Mais60 & Média & $e^{2}$ e valor de $p$ \\
\hline & Minus30 & Standard & Plus30 & Plus60 & Mean & $e f^{2}$ and $p$ value \\
\hline & \multicolumn{6}{|c|}{ MSV (kg/ha) } \\
\hline & \multicolumn{6}{|c|}{$G D M(k g / h a)$} \\
\hline Mombaça & 156 & 790 & 651 & 1010 & $644 A^{3}$ & \\
\hline Tanzânia & 198 & 337 & 312 & 874 & 437B & \\
\hline \multirow[t]{2}{*}{ Média (Mean) } & 176 & 535 & 481 & 942 & $\mathrm{CV}^{4}=58,8 \%$ & ef $L, p<0,01$ \\
\hline & \multicolumn{6}{|c|}{$\begin{array}{c}\text { \% MSV perdida por pisoteio em relação a MSV em pré-pastejo } \\
\% \text { GDM losses by trampling to GDM before grazing }\end{array}$} \\
\hline Mombaça & 2,0 & 14,5 & 7,8 & 10,3 & 8,7 & \\
\hline Tanzânia & 4,7 & 5,1 & 4,1 & 12,6 & 6,7 & \\
\hline \multirow[t]{2}{*}{ Média (Mean) } & 3,4 & 9,8 & 6,0 & 11,5 & $C V=108 \%$ & ef $L, p<0,05$ \\
\hline & \multicolumn{6}{|c|}{$\begin{array}{l}\% \text { de lâminas foliares } \\
\text { Leaf blade \% }\end{array}$} \\
\hline Mombaça & 39,72 & 61,81 & 45,01 & 53,46 & $50,00 \mathrm{~A}$ & \\
\hline Tanzânia & 46,24 & 36,92 & 39,46 & 39,80 & $40,44 \mathrm{~B}$ & \\
\hline \multirow[t]{2}{*}{ Média (Mean) } & 42,79 & 49,36 & 42,23 & 46,63 & $C V=34,4 \%$ & $\mathrm{~ns}$ \\
\hline & \multicolumn{6}{|c|}{$\begin{array}{l}\% \text { de colmos } \\
\text { Stem } \%\end{array}$} \\
\hline Mombaça & 38,05 & 38,18 & 54,98 & 46,53 & $44,41 \mathrm{~B}$ & \\
\hline Tanzânia & 53,75 & 63,08 & 60,53 & 60,19 & $59,55 \mathrm{~A}$ & \\
\hline \multirow[t]{2}{*}{ Média (Mean) } & 45,44 & 50,63 & 57,36 & 53,36 & $\mathrm{CV}=24.1 \%$ & ef $L, p<0,05$ \\
\hline & \multicolumn{6}{|c|}{$\begin{array}{l}\text { Relação lâmina/colmo } \\
\text { Leafblade/stem ratio }\end{array}$} \\
\hline Mombaça & 0,76 & 1,85 & 0,78 & 1,24 & $1,18 \mathrm{~A}$ & efC, $p<0,01$ \\
\hline Tanzânia & 0,95 & 0,62 & 0,73 & 0,79 & $0,77 \mathrm{~B}$ & ns \\
\hline Média (Mean) & 0,85 & 1,24 & 0,75 & 1,01 & $\mathrm{CV}=59,9 \%$ & \\
\hline
\end{tabular}

${ }^{1} \mathrm{~A}$ dose de NPK "Padrão" corresponde a 145, 21,6 e $180 \mathrm{~kg} / \mathrm{ha}$ de $\mathrm{N}, \mathrm{P}_{2} \mathrm{O}_{5}$ e $\mathrm{K}_{2} \mathrm{O}$, respectivamente. Menos 30 , Mais30 e Mais60 correspondem ao decréscimo em $30 \%$ e ao acréscimo em 30 e $60 \%$ da dose "Padrão", respectivamente ("Standard" correponded to the aplication of 145, 21.6 and $180 \mathrm{~kg} / \mathrm{ha}$ of $\mathrm{N}, \mathrm{P}_{2} \mathrm{O}_{5}$ and $\mathrm{K}_{2} \mathrm{O}$, respectively (Minus30, Plus 30 and Plus60 corresponded to the reduction of $30 \%$ and increase of 30 and $60 \%$ in relation to the "Standard" dose, respectively).

2 Efeito da adubação: ef $L=$ efeito linear, ef $Q=$ =efeito quadrático, ef $C=$ efeito cúbico e ns=não significativo (Effect of fertilization: ef $L=l i n e a r$ effect, ef $Q=q u a d r a t i c ~ e f f e c t$, ef $C=c u b i c$ effect and $n s=n o n$ significant).

${ }^{3}$ Letras diferentes na mesma coluna diferem $(p<0,05)$ pelo Teste de Tukey (Different letters in the same column differ $(p<0.05)$ by the test of Tukey).

${ }^{4} \mathrm{CV}=$ coeficiente de variação ( $\mathrm{CV}=$ coefficient of variation).

A taxa de lotação estimada do cv. Tanzânia com a aplicação de doses crescentes de NPK aumentou de 2,6 a 8,8 UA/ha, nas doses "menos 30" a "mais 60", respectivamente. $\mathrm{O} \mathrm{cv}$. Mombaça apresentou taxa de lotação estimada considerada alta mesmo nas menores doses de NPK testadas, com variação de 7,5 a 10,7 UA/ha nas doses "menos 30" e "mais 60", respectivamente.

\section{Conclusões}

Doses crescentes de NPK resultaram em maiores produções de MSV nos cultivares Tanzânia e
Mombaça, o que está associado ao aumento do peso médio dos perfilhos.

O percentual de MS senescida em relação à MST da parte aérea das plantas foi maior no cv. Tanzânia do que no cv. Mombaça. O aumento das doses de NPK aplicadas resultou em aumento das perdas de MSV por pisoteio.

$\mathrm{O}$ cv. Mombaça apresentou maior potencial de resposta à adubação com NPK, admitindo taxas de lotação estimada mais elevadas do que o cv. Tanzânia, o que permite sugerir a sua aptidão para sistemas mais intensivos de produção de ruminantes em pastagens, apesar de exigir manejo mais criterioso. 


\section{Literatura Citada}

ALCÂNTARA, P.B.; PEDRO Jr., M.J.; DONZELLI, P.L. Zoneamento edafoclimático de plantas forrageiras. In: SIMPÓSIO SOBRE ECOSSISTEMAS DE PASTAGENS, 2., 1993, Jaboticabal. Anais... Jaboticabal: Fundação Universidade Estadual Paulista, 1993. p.1-16.

CECATO, U.; MACHADO, A.O.; MARTINS, E.N. et al. Avaliação da produção e de algumas características da rebrota de cultivares e de acessos de Panicum maximum Jacq. sob duas alturas de corte. Revista Brasileira de Zootecnia, v.29, n.3, p.660-668, 2000.

CORRÊA, L.A. Pastejo rotacionado para produção de bovinos de corte. In: SIMPÓSIO DE FORRAGICULTURA E PASTAGENS: Temas em evidência, 2000, Lavras. Anais... Lavras: Universidade Federal de Lavras, 2000. p.149-177.

EUCLIDES, V.P.B.; THIAGO, L.R.L.S.; MACEDO, C.M.M. et al. Consumo voluntário de forragem de três cultivares de Panicum maximum sob pastejo. Revista Brasileira de Zootecnia, v.28, n.6, p.1177-1185, 1999.

FORNI, S.; MICHEL FILHO, L.; FAVORETTO, V. et al. Efeito de diferentes doses de adubação com NPK sobre a produção, qualidade e estrutura das cultivares Tanzânia e Mombaça de Panicum maximum Jacq. In: REUNIÃO ANUAL DA SOCIEDADE BRASILEIRA DE ZOOTECNIA, 37., 2000, Viçosa, MG. Anais... São Paulo: SBZ/Gmosis, [2000] 17 par. CD-ROM. Forragicultura. Apresentação oral. FOR-702.

GOMIDE, J.A. Aspectos biológicos e econômicos da adubação de pastagens. In: SIMPÓSIO SOBRE ECOSSISTEMAS DE PASTAGENS, 1989, Jaboticabal. Anais... Jaboticabal: Fundação Universidade Estadual Paulista, 1989. p.237-270.

HERLING, V.R. Efeitos de níveis de nitrogênio sobre algumas características fisiológicas e qualitativas dos cultivares colonião e centenário de Panicum maximum Jacq. Jaboticabal: Universidade Estadual Paulista, 1995. 125p. Tese (Doutorado em Zootecnia) - Universidade Estadual Paulista, 1995.

HUMPHREYS, L.R. Tropical pasture utilization. Cambridge: Cambridge University Press, 1991. 206p.

JANK, L. Melhoramento e seleção de variedades de Panicum maximum. In: SIMPÓSIO SOBRE MANEJO DA PASTAGEM, 12., Piracicaba, 1995. Anais... Piracicaba: Fundação de Estudos Agrários Luiz de Queiroz, 1995. p.21-58.

LOBATO, E.; KORNELIUS, E.; SANZONOWICZ, C. Adubação fosfatada em pastagens. In: PEIXOTO, A.M.; MOURA, J.C.; FARIA, V.P. (Eds.) Pastagens - fundamentos da exploração racional. 2.ed. Piracicaba: Fundação Estudos Agrários Luiz de Queiroz, 1994. p.155-188.

MARASCHIN, E.G. Perdas de forragem sob pastejo. In: SIMPÓSIO SOBRE ECOSSISTEMAS DE PASTAGENS, 2., 1993, Jaboticabal, Anais... Jaboticabal: Fundação Universidade Estadual Paulista, 1993. p.166-190.

MAZZANTI, A.; LEMAIRE, G. Effect of nitrogen fertilization on herbage production of tall fescue swards continuously grazed by sheep. 2. Consumption and efficiency of herbage utilization. Grass and Forage Science, v.49, n.3, p.352359,1994

MONTEIRO, F.A. Nutrição mineral e adubação. In: SIMPÓSIO SOBRE MANEJO DA PASTAGEM, 12, 1995, Piracicaba. Anais... Piracicaba: Fundação Estudos Agrários Luiz de Queiroz, 1995. p.219-244.
MONTEIRO, F.A.; LIMA, S.A.A.; WERNER, J.C. et al. Adubação potássica em leguminosas e em capim-colonião (Panicum maximum Jacq.) adubado com níveis de nitrogênio ou consorciado com leguminosas. Boletim da Indústria Animal, v.37, n.1, p.127-148, 1980.

MONTEIRO, F.A.; WERNER, J.C. Efeitos das adubações nitrogenadas e fosfatadas em capim-colonião, na formação e em pasto estabelecido. Boletim da Indústria Animal, v.34, n.1, p.91-101, 1977.

NABINGER, C. Eficiência do uso de pastagens: disponibilidade e perdas de forragem. In: SIMPÓSIO SOBRE MANEJO DA PASTAGEM: fundamentos do pastejo rotacionado, 14., 1997, Piracicaba. Anais... Piracicaba: Fundação Estudos Agrários Luiz de Queiroz, 1997. p.231-251.

PIETERSE, P.A.; RETHMAN, N.F.G.; Van BOSCH, J. Production, water use efficiency and quality of four cultivars of Panicum maximum at different levels of nitrogen fertilization. Tropical Grasslands, v.31, n.2, p.117-123, 1997.

ROSSETO, F.A.A. Desempenho agronômico de pastagens de capim elefante cv. Guaçu (Pennisetum purpureum Schum.) e capim Tanzânia (Panicum maximum Jacq.) em sistemas de produção de leite. Piracicaba: Escola Superior de Agricultura Luiz de Queiroz, 2000. 173p. Dissertação (Mestrado em Agronomia) - Escola Superior de Agricultura Luiz de Queiroz, 2000.

SANTOS, P.M.; CORSI, M.; BALSALOBRE, M.A.A. Efeito da freqüência de pastejo e da época do ano sobre a produção e a qualidade em Panicum maximum cvs. Tanzânia e Mombaça. Revista Brasileira de Zootecnia, v.28, n.2, p.244-249, 1999.

SHAW, N.H.; JONES, R.M.; EDYE, L.A. et al. Developing and testing new pastures. In: SHAW, N.H.; BRYAN, W.W. (Eds.) Tropical pasture research: principles and methods. Hurley: CAB, 1976. p.175-193. (Bulletin 51)

SILVA, D.J. Análise de alimentos: métodos químicos e biológicos. 2.ed. Viçosa, MG: Universidade Federal de Viçosa, 1998. $166 \mathrm{p}$.

SISTI, C.P.J.; HERLING, V.R.; LUZ, P.H.C. et al. Efeito de ofertas de forragem e de períodos de descanso sobre a produção, perdas e resíduo de matéria seca do capim mombaça (Panicum maximum Jacq.) In: REUNIÃO ANUAL DA SOCIEDADE BRASILEIRA DE ZOOTECNIA, 36., 1999, Porto Alegre. Anais... São Paulo: SBZ/Gmosis, \{1999\} 17 par. CD-ROM. Forragicultura. Avaliação com animais. FOR-10.

STATISTICAL ANALYSES SYSTEM. SAS User's guide: Statistic. 6.ed. Cary: 1996. 956p.

TEIXEIRA, E.I.; MATTOS, W.R.S.; CAMARGO, A.C. et al. Avaliação da produção e utilização de uma pastagem de capim Tobiatã (Panicum maximum cv. Tobiatã) sob pastejo rotacionado. Scientia Agricola, v.56, n.2, p.349-355, 1999

VALLENTINE, J.F. Grazing management. New York: Academic Press, 1990. 533p.

VICENTE-CHANDLER, J. Fertilization of humid tropical grasslands. In: MAYS, D.A. (Ed.) Forage fertilization. Madison:ASA-CSA-SSSA, 1974. p.277-300.

WERNER, J.C. Adubação de pastagens. Nova Odessa: Instituto de Zootecnia. 1986. 49p. (Boletim técnico 18.)

Recebido em: 20/06/01 Aceito em: 15/03/02 